\title{
Rarefied gas flow in pressure and vacuum measurements
}

\author{
Jeerasak Pitakarnnop, Rugkanawan Wongpithayadisai
}

National Institute of Metrology, Prathumthani, Thailand

\begin{abstract}
Flows of a gas through the piston-cylinder gap of a gas-operated pressure balance and in a general vacuum system have one aspect in common, namely that the gas is rarefied due, respectively, to the small dimensions and the low pressure. The flows in both systems could be characterised as being in either slip-flow or transition regimes. Therefore, fundamental research of flow in these regimes is useful for both pressure and vacuum metrology, especially for the gas-operated pressure balance where a continuum viscous flow model is widely used for determining the effective area of the pressure balance. The consideration of gas flow using the most suitable assumption would improve the accuracy of such a calculation. Moreover, knowledge about rarefied gas flow will enable gas behaviour in vacuum and low-flow leak detection systems to be predicted. This paper provides useful information about rarefied gas flow in both slip-flow and transition regimes.
\end{abstract}

\section{Section: RESEARCH PAPER}

Keywords: Rarefied Gas; Slip-Flow; Kinetic Theory

Citation: Jeerasak Pitakarnnop, Rugkanawan Wongpitayadisai, Rarefied gas flow in pressure and vacuum measurements, Acta IMEKO, vol. 3, no. 2, article 14, June 2014, identifier: IMEKO-ACTA-03 (2014)-02-14

Editor: Paolo Carbone, University of Perugia

Received February $14^{\text {th }}, 2014$; In final form May 25 ${ }^{\text {th }}, 2014$; Published June 2014

Copyright: (C) 2014 IMEKO. This is an open-access article distributed under the terms of the Creative Commons Attribution 3.0 License, which permits unrestricted use, distribution, and reproduction in any medium, provided the original author and source are credited

Funding: (none reported)

Corresponding author: Jeerasak Pitakarnnop, e-mail: Jeerasak@nimt.or.th

\section{INTRODUCTION}

Characteristic scale, $L_{C}$, and pressure, $p$, are the two main factors that characterize the flow regime in a gas-operated system. The micro-scale gap between the piston and cylinder of a pressure balance and the ultra-low pressure in a vacuum system will reduce the large number of gas molecules and cause the gas to be rarefied. Consequently, due to the smaller number of gas molecules, flow behaviour will be different from general gas where the number of gas molecules is large enough to consider the gas as a continuum medium. The continuum medium assumption is not valid for the aforementioned cases if the flow behaves as slip, transition, or free molecular flow. The flow regime is characterized according to its Knudsen number, $\mathrm{Kn}$ :

$$
\mathrm{Kn}=\frac{\lambda}{L_{C}}
$$

where $\lambda$ is the molecular mean free path and $L_{C}$ is the characteristic scale of the gas flow. With respect to the value of the Knudsen number, there are four distinct regimes as shown in Figure 1. When $\mathrm{Kn}$ is very small, there are enough molecules for the gas to be considered as in a continuum regime. Slip-flow and other effects, such as a temperature jump at a solid surface, start to appear at values of $\mathrm{Kn}$ greater than 0.001 and become dominant at around 0.01, where the slip-flow regime begins. As the gas becomes more and more rarefied, its flow is characterized as being in the transition and free molecular regimes, when $\mathrm{Kn}$ reaches 0.1 and 10, respectively. In order to predict gas behaviour accurately, it is necessary to know its flow regime. Using incorrect assumptions can lead to large errors.

As well as the Knudsen number, the rarefaction parameter $\delta$ is another quantity that is also used to describe the flow regime and is defined as:

$\delta=\frac{\sqrt{\pi}}{2} \frac{L_{C}}{\lambda}=\frac{\sqrt{\pi}}{2} \frac{1}{\mathrm{Kn}}$

The molecular mean free path was not directly measured. In this paper, it is estimated using Maxwellian theory as:

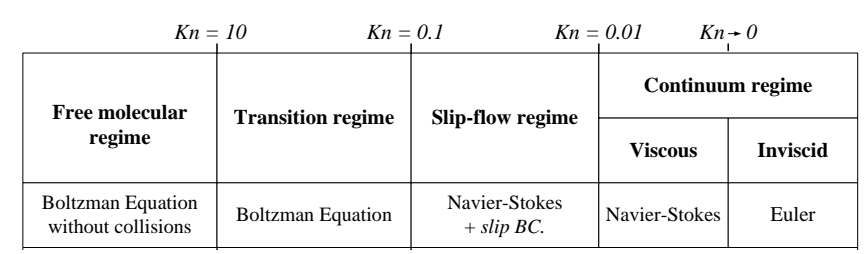

Figure 1. Classification of gas flow regime [1]. 


$$
\lambda=\frac{\sqrt{\pi}}{2} \frac{\mu \tilde{v}}{p}
$$

where $\mu$ is the viscosity at temperature $T$ and $\tilde{\mathrm{v}}=\sqrt{2 \mathrm{RT}}$ is the most probable molecular velocity. From the above equations, the Knudsen number as a function of the pressure of gas flowing through a piston-cylinder gap in gauge and absolute modes, and through ISO-standard tube, is plotted in Figure 2.

According to Figure 1, with the pressure balance operating in absolute mode (dashed line), flows through a piston-cylinder gap of $0.3 \mu \mathrm{m}, 0.6 \mu \mathrm{m}$, and $0.9 \mu \mathrm{m}$ are in the transition and free molecular flow regimes. Even in gauge mode (strong solid line), the gas is rarefied enough to be characterised as being in either a slip or transition regime. It would be inappropriate to use Dadson's theory [2], which is based on continuum flow assumptions, to calculate the effective area of a piston-cylinder assembly under these conditions. This theory has therefore been modified to produce more accurate results [3][4][5]. This paper proposes an alternative method, especially in the slip-flow regime where continuum assumptions are still valid with special consideration at the solid surface. This method, in which a slip boundary is applied at the surface, is useful for simulating flow through a piston-cylinder gap using commercial CFD software.

Flow in a vacuum system could be in any regime from continuum to free molecular, depending on the pressure and the dimensions of the gas container and passages. For conventional ISO tube (solid line), rarefied gas effects start once the pressure falls below $1000 \mathrm{~Pa}$. At this point, conventional continuum theory begins to break down and Navier-Stokes equations are no longer valid.

The objective of this paper is to demonstrate the results of slip-flow and kinetic BGK (Bhatnagar-Gross-Krook) models in both the slip-flow and transition regimes, the most common ones encountered in pressure and vacuum metrology. Slip-flow equations, which extend the validity of the Navier-Stokes model, are detailed. These equations have been rigorously validated for microchannels [8]. The presented models are simpler than the kinetic ones, yet still provide quite accurate results within slip-flow regimes.

\section{SLIP-FLOW}

The slip-flow regime is a slightly rarefied one, which could occur either in gas flows through the piston-cylinder gap and or in vacuum systems, as shown in Figure 2. It typically corresponds to a Knudsen number ranging between 0.01 and 0.1 , easily reached for flow either through a micrometer scale gap in a pressure balance operating in gauge mode under

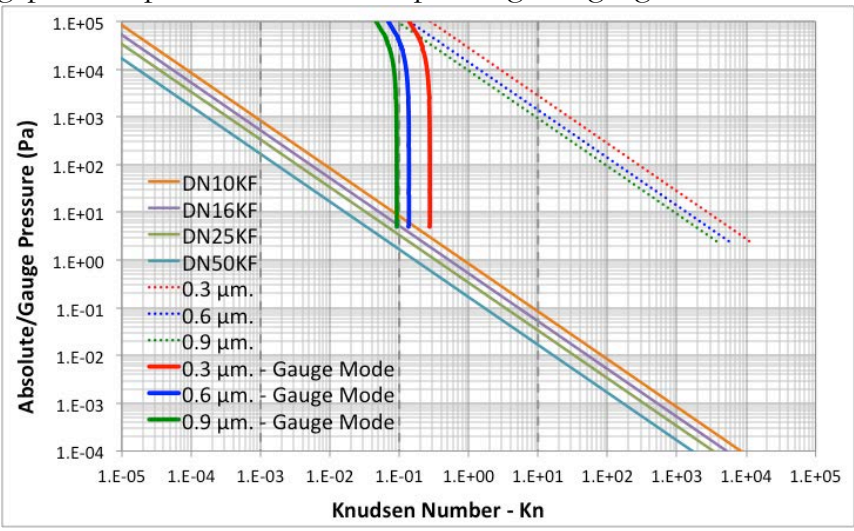

Figure 2. Pressure versus $\mathrm{Kn}$ for usual gas flow through different pistoncylinder gaps and vacuum tubes for $\mathrm{N}_{2}$ at $20^{\circ} \mathrm{C}$. standard conditions or in rough vacuum. The Knudsen layer plays a fundamental role in the slip-flow regime. This thin layer, one or two molecular mean free paths in thickness, is a region of local non-equilibrium, observed in any gas flow near a surface. In non-rarefied flow, the Knudsen layer is too thin to have any significant influence but, in the slip-flow regime, it needs to be considered [6].

Although the Navier-Stokes equations are not valid in the Knudsen layer, due to non-linear stress/strain-rate behaviour within it [7], their use with appropriate boundary velocity slip and temperature jump conditions can provide an accurate prediction of mass flow rate [8]. The slip-flow condition was originally proposed by Maxwell and has since been developed up to the second order. Several models have been proposed, most of similar form but differing slightly in the coefficients used. If isothermal flow is assumed, a general second order slipflow model is derived as:

$u_{\text {sip }}=u_{s}-u_{w}=\left.\frac{2-\alpha}{\alpha} A_{\alpha} \lambda \frac{\partial u}{\partial n}\right|_{\text {well }}-\left.A_{\beta} \lambda^{2} \frac{\partial^{2} u}{\partial n^{2}}\right|_{\text {well }}$

where $u_{s i p}$ is the slip velocity, $u_{s}$ is the flow velocity at the wall, and $u_{w}$ is the velocity of the wall, with its normal direction noted as $n$. The mean free path of the molecules is $\lambda$ and $a$ is the tangential momentum accommodation coefficient, equal to unity for perfectly diffuse molecular reflection and equal to zero for purely specular reflection. $A_{a}$ and $A_{\beta}$ are the first and second order dimensionless coefficients, respectively. In Maxwell's model, $A_{a}$ was taken as being equal to unity which overestimates the velocity at the wall but leads to a good prediction of gas velocity outside the Knudsen layer. Example values of $A_{a}$ and $A_{\beta}$ proposed in the literature are given in Table 1.

To determine pressure distribution along piston and cylinder surfaces or flow through vacuum systems, the boundary equation (4) is applied to Navier-Stokes equations. These equations could be solved analytically for flow through simple geometries, whereas flow within a more complicated model requires a numerical calculation. Normally, commercial CFD (Computational Fluid Dynamics) software such as ANSYS FLUENT enables slip boundary conditions to be input at a boundary surface. Methods to apply the boundary conditions in CFD software have been presented in the literature [6] [9].

Gas flow through a piston-cylinder gap is considered as a flow between two infinite parallel plates (or slabs) in the analysis, as the gap between the piston and cylinder of a pressure balance is very small in comparison to the radius of piston. After applying the slip coefficient to the Navier-Stokes equations, a reduced flow rate for slab flow is derived in terms of the rarefaction parameter as:

$G_{p}=\frac{\delta}{6}+\frac{2-\alpha}{\alpha} A_{\alpha} \sqrt{\pi}-A_{\beta} \frac{\pi}{\delta}$

Since the rarefaction parameter in equation (5) depends on pressure, the reduced flow rate $G_{p}$ and the pressure distribution along the slab need to be computed iteratively. The equation of pressure distribution along a piston and cylinder was derived by

Table 1. Example values of the dimensionless coefficients

\begin{tabular}{lcc}
\hline Author, Year & $\boldsymbol{A}_{\boldsymbol{\alpha}}$ & $\boldsymbol{A}_{\boldsymbol{B}}$ \\
\hline Maxwell, 1879 & 1.0000 & 0.0000 \\
Cercignani, 1964 & 1.1466 & 0.9756 \\
Deissler, 1964 & 1.0000 & 1.1250 \\
Hadjiconstantinou, 2003 & 1.1466 & 0.6470 \\
\hline
\end{tabular}


Priruenrom [10] as:

$$
p(z)=p_{1}-\left(p_{1}-p_{2}\right) \int_{0}^{z} \frac{1}{h^{2} G_{p}(z)} c z / \int_{0} \frac{1}{h^{2} G_{p}(z)} c z
$$

where $p_{1}$ is the applied pressure at the bottom of piston, $p_{2}$ is the pressure above the piston, $z$ is the axial coordinate along the piston and cylinder, and $l$ is the piston-cylinder overlapped length. Further information on how to determine an effective area and a pressure distortion coefficient using the above equation is presented in her thesis.

The previously-discussed slip-flow methods could also be used to predict gas flow through a vacuum piping system, the only difference being the flow passage's cross-sectional geometry, which is normally circular. The reduced flow rate for slip-flow through a tube is calculated as:

$$
\mathrm{G}_{p}=\frac{\delta}{4}+\frac{2-\alpha}{\alpha} \boldsymbol{A}_{\alpha} \frac{\sqrt{\pi}}{2}-A_{\beta} \frac{\pi}{4 \delta}
$$

A flow parameter that is of common interest is mass flow rate through the passage. This can be calculated from the reduced flow rate as follows [11][12]:

For the slab flow,

$$
\dot{m}_{s,}=\frac{A H}{\tilde{v}} G_{p}^{g} \frac{d p}{c z}
$$

where $A$ is the cross-sectional area of the channel and $H$ is the height of the slab.

For flow through a circular tube,

$$
\dot{m}_{b b}=\frac{\pi R^{\beta}}{\tilde{v}} G_{p}^{b} \frac{d p}{d z}
$$

where $R$ is the radius of the tube.

\section{TRANSITION FLOW}

As discussed in the previous section, slip-flow models are limited to use within the slip-flow regime whereas, within the transition regime, kinetic gas theory must be used. Solutions based on this theory are valid throughout the whole range of the Knudsen number from the free molecular, through the transition, and up to the slip and hydrodynamic regimes. In this paper, the BGK (one kinetic gas model) is chosen and the linearised BGK model is solved numerically by DVM (Discrete Velocity Method) to determine flow behaviour. Previous research work [8] has demonstrated that fully-developed isothermal pressure-driven flows are accurately predictable by kinetic models, such as the linearised BGK equation. Figure 3 shows a comparison between measurement results and those from the BGK model.

The gas flow rate measurements were performed at the inlet

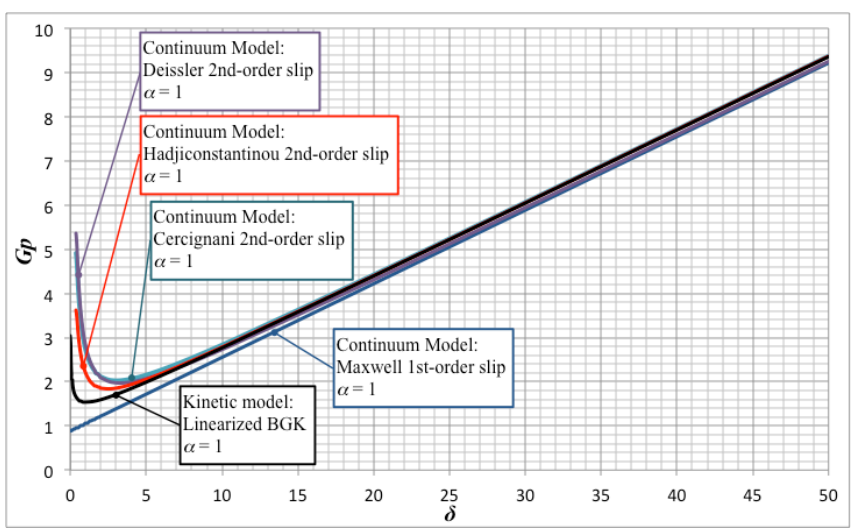

Figure 4. Reduced flow rate $\left(G_{p}\right)$ versus Rarefaction parameter $(\delta)$ of flow through slab at diffuse reflection $(\alpha=1)$. and the outlet $(\square)$ of a series of rectangular microchannels whose aspect ratio was approximately 0.1 . The reduced flow rate $G_{p}$ is plotted in terms of the rarefaction parameter $\delta$. Measurement results are in good agreement with the kinetic model based on the linearised BGK equation, with $a=1$ or diffuse reflection where gas molecules lose all their tangential momentum to a wall during their collisions. Details of the investigation are described in the literature.

\section{RESULTS}

Results of the slip-flow model for slab flow are shown in Figure 4, where the reduced flow rates $G_{p}$, from equation (5) for each model presented in Table 1 , are plotted in terms of the rarefaction parameter $\delta$. The result from the BGK model is plotted as a benchmark, against which the results from the other models can be compared. All results are in very good agreement in the slip-flow regime $(\delta>8.86$ or $\mathrm{Kn}<0.1)$, except the first order slip-flow model significantly deviates from the others near the upper limit of the slip-flow regime. A difference of up to $8.5 \%$ is observed at $\delta=9$ when compared with the result from the BGK method. The Hadjiconstantinou equation yields the closest result to BGK method, with less than a $0.9 \%$ difference observed for the entire slip-flow regime.

An overview showing the region from the slip-flow regime to the free molecular regime is given in Figure 5. To help focus attention on the transition regime, the rarefaction parameter $\delta$ is plotted on a logarithmic scale.

The result of the BGK model when the tangential accommodation $a$ is equal to 0.9 is plotted to demonstrate the trend in the results when the gas-surface interaction is no longer considered to be a purely diffuse collision. Moreover, results of the BGK model from Cercignani \& Pagani (ם) [13], Lo \& Loyalka $(\Delta)$ [14], and Loyalka et al. (o) [15] are also plotted to support the results obtained. Any differences between the results of the various BGK models are insignificant throughout all regimes, whereas the results from all slip-flow models fail to predict rarefied gas flow in both the transition and molecular flow regimes.

For flow within a circular tube, the trend of the results shown in Figure 6 does not differ much from the previous slab flow case. The results from the BGK model at $a=1$ are in very good agreement with those of Cercignani \& Sernagiotto [16], Sharipov $(\Delta)$, and Loyalka \& Hamoodi (o), using a BGK model [17], a Shakov model, and a Numerically Solved Boltzmann Equation [18] respectively.

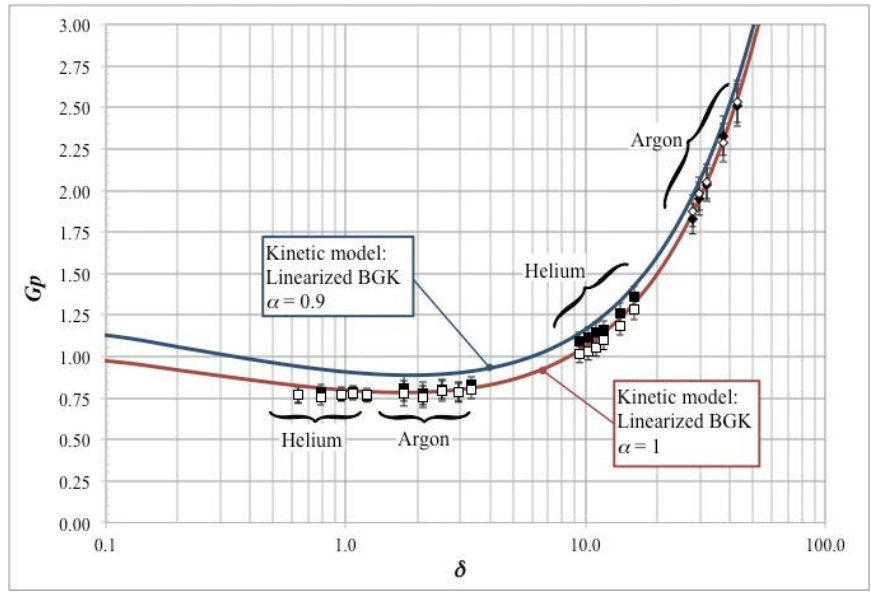

Figure 3. Reduced flow rate $\left(G_{p}\right)$ versus rarefaction parameter $(\delta)$ of flow through rectangular channel (aspect ratio $\approx 0.1$ ) [7]. 


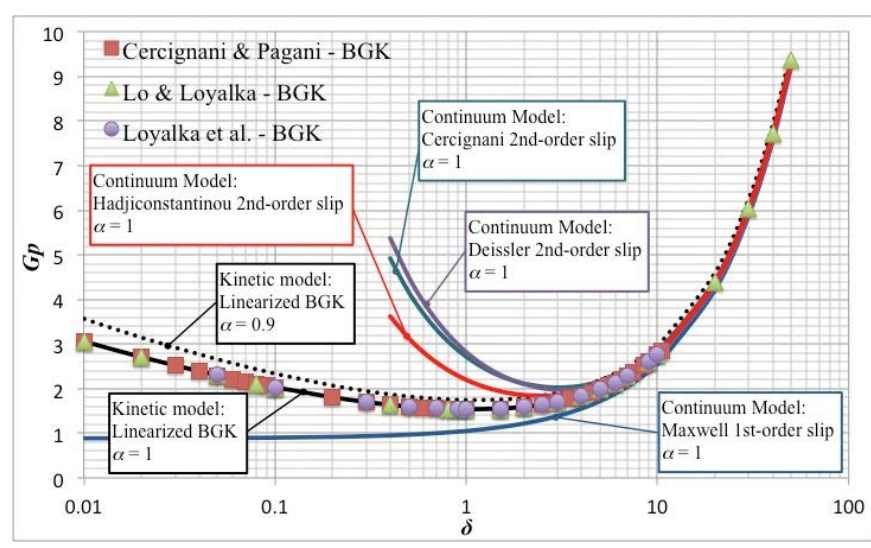

Figure 5. Reduced flow rate $\left(G_{p}\right)$ versus rarefaction parameter $(\delta)$ of flow through slab $(\alpha=0.9,1)$.

As in the slab flow case, all slip-flow equations break down in the transition and free molecular regimes. However, within the slip-flow regime itself, the boundary equation in conjunction with the Navier-Stokes equation is the preferred method to solve for bulk flow, due to the less complex calculations and the lower required resources. For simple geometry cases, it is possible to obtain an exact solution.

\section{DISCUSSIONS AND CONCLUSION}

General equations to determine the flow rate of rarefied gas through both slab and tube, based on Navier-Stoke equations in conjunction with slip boundary conditions, have been developed. The results of these slip-flow models have been compared with those from kinetic theory using a BGK model, the results of which have been obtained by a numerical method using a DVM (Discrete Velocity Method) scheme. Slip-flow models require lower computational resources, but their performance is limited. They provide a reliable result within the continuum and slip-flow regimes but fail to predict rarefied gas flow in both the transition and free molecular regimes.

Since the slip-flow method is easier both to handle and apply in commercial CFD (Computational Fluid Dynamic) software for solving complex problems, it is still a valuable approach. It can be used as an alternative method to determine gas flow within a piston-cylinder gap in a slip-flow regime. Moreover, such a method can also provide a precise prediction of flow rate through the piping of a vacuum system operating within the slip-flow regime.

\section{ACKNOWLEDGMENT}

Several years ago, Prof. Stéphane Colin, Prof. Lucien Baldas, Prof. Sandrine Geoffroy, and their colleagues at the institut clément ader opened a door to the world of rarefied gas dynamics to the first author, giving him the opportunity to join their research. A few years later, the chance to meet Prof. Dimitris Valougeorgis and colleagues at the University of Thessaly brought him a key to solve kinetic theory of gases. He is forever grateful to them for their kind support in knowledge and mind.

\section{REFERENCES}

[1] D. Valougeorgis, "Solution of vacuum flows via kinetic theory," 51 It IUVSTA Workshop on modern problems and capability of vacuum gas dynamics, 2007.

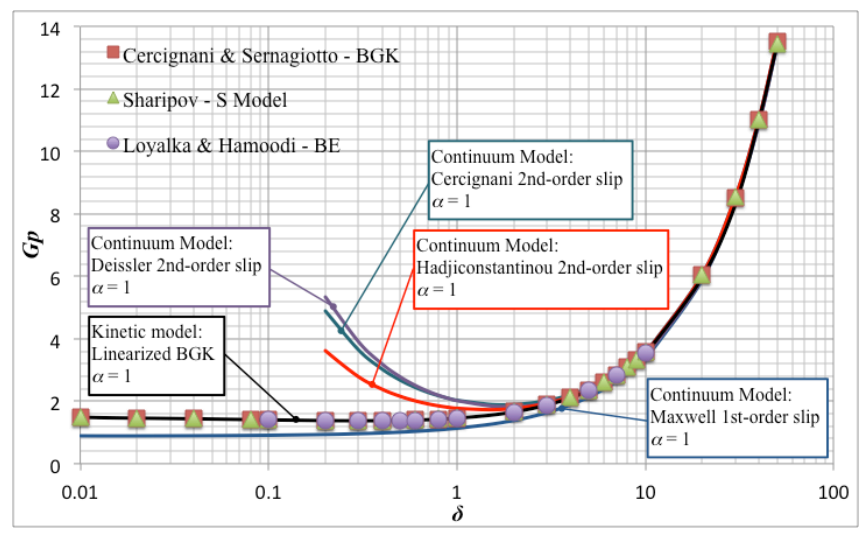

Figure 6 . Reduced flow rate $\left(G_{p}\right)$ versus rarefaction parameter $(\delta)$ of flow through circular tube at diffuse reflection $(\alpha=1)$.

[2] R. S. Dadson, S. L. Lewis and G. N. Peggs, The pressure balance: Theory and practice. London, HMSO, 1982.

[3] C. M. Sutton, "The effective area of a pressure balance at low pressures,” J. Phys. E: Sci. Instrum., vol. 13, pp. 857-859, 1980.

[4] J. W. Schmidt, B. E. Welch, and C. D. Ehrlich, "Operational mode and gas species effects on rotational drag in pneumatic dead weight pressure gauges," Meas. Sci. Technol., vol. 4, pp. 26-34, 1993.

[5] J. W. Schmidt, S. A. Tison, and C. D. Ehrlich, "Model for drag forces in the crevice of piston gauges in the viscous-flow and molecular flow regimes," Metrologia, vol. 36, pp. 565-570, 1999.

[6] J. Pitakarnnop, S. Geoffroy, S. Colin and L. Baldas, "Slip flow in triangular and trapezoidal microchannels," International Journal of Heat and Technology, vol. 26, no. 1 pp. 167-174, 2008.

[7] D. A. Lockerby, J. M. Reese, and M. A. Gallis, "Capturing the Knudsen layer in continuum-fluid models of nonequilibriun gas flows," AIAA Journal, vol. 43, no. 6 pp. 1391-1393, 2005.

[8] J. Pitakarnnop, S. Varoutis, D. Valougeorgis, S. Geoffroy, L. Baldas and S. Colin, "A novel experimental setup for gas microflows," Microfluid Nanofluid, vol. 8, no. 1 pp. 57-72, 2010.

[9] J. Pitakarnnop, Analyse expérimentale et simulation numérique d'écoulements raréfiés de gaz simple et de mélanges gazeux dans les microcanaux, Université de Toulouse, 2009.

[10] T. Priruenrom, Development of pressure balance for absolute pressure measurement in gases up to $7 \mathrm{MPa}$, Clausthal University of Technology, 2011.

[11] F. Sharipov and V. Seleznev, "Data on internal rarefied gas flows," J. Phys. Chem. Ref. Data, vol. 27, no. 3 pp. 657-706, 1998.

[12] S. Varoutis et al, "Computational and experimental study of gas flows through long channels of various cross sections in the whole range of the Knudsen number," J. Vac. Sci. Technol. A, vol. 27, no. 1 pp. 89-100, 2009.

[13] C. Cercignani and C. D. Pagani, "Variational approach to boundary-value problems in kinetic theory," Phys. Fluids, vol. 9 , no. 6, pp. 1167-1173, 1966.

[14] S. S. Lo and S. K. Loyalka, "An efficient computation of nearcontinuum rarefied gas flows,” J. Appl. Math. Phys. (ZAMP), vol. 33 , no. 3 pp. 419-424, 1982.

[15] S. K. Loyalka, N. Petrellis and T. S. Storvick, "Some exact numerical results for the BGK model: Couette, Poiseuille and thermal creep flow between parallel plates," J. Appl. Math. Phys. (ZAMP), vol. 30, no. 3 pp. 514-521, 1979.

[16] C. Cercignani and F. Sernagiotto, "Cylindrical Poiseuille flow of a rarefied gas,” Phys. Fluids, vol. 9, no. 1, pp. 40-44, 1966.

[17] F. Sharipov, "Rarefied gas flow through a long tube at any temperature ratio," J. Vac. Sci. Technol. A, vol. 14, no. 4, pp. 2627-2635, 1996.

[18] C. Cercignani and S. A. Hamoodi, "Poiseuille flow of a rarefied gas in a cylindrical tube: solution of linearized Boltzmann equation," Phys. Fluids, vol. 2, no. 11, pp. 2061-2065, 1990. 